\title{
Accuracy of p16/Ki-67 dual staining for improved cervical cancer risk stratification in women aged $\leq 30$ years with $\mathrm{hr}-\mathrm{HPV}$ positive
}

\section{Xingmin Wang ( $\nabla$ wangxingmin@163.com)}

Liuzhou Maternity and Child Care Health

\section{Xindan Wang}

Liuzhou Maternity and Child Care Health

\section{Ping Wu}

Chengdu First People's Hospital

\section{Jinjian Fu}

Liuzhou Maternity and Child health care hospital

\section{Guixian Yi}

Dongguan Human Hospital of Chinese Medicine

Jianhua Xu

Shunde Hospital of Guangzhou University of Chinese Medicine

\section{Research article}

Keywords: Cervical intraepithelial neoplasia, Human papillomavirus, p16/Ki67 dual staining, Screening

Posted Date: October 13th, 2020

DOI: https://doi.org/10.21203/rs.3.rs-91126/v1

License: (c) (i) This work is licensed under a Creative Commons Attribution 4.0 International License. Read Full License 


\section{Abstract}

Background: Cervical cancer (CC) is the most common malignancy inwomen on the earth. Cervical cancer usually develops from cervical intraepithelial neoplasia (CIN) grade 1 or above. Early detection of CIN1 or above precancerous lesion can effective control of cervical cancer incidence. The goal of this study was to evaluate the accuracy of p16/Ki67 dual staining in triaging hr-HPV positive population aged $\leq 30$ years.

Methods: A total of 336 women with an average age of 27.8 years old were included in this study. Liquid based cytology (LBC) samples were detected by p16/Ki67 immunocytochemical dual staining, liquidbased cytology, high-risk human papillomavirus (hr-HPV) and HPV 16/18 test. Diagnosis of each method was verified by histopathological test. Sensitivity, specificity, positive predictive value (PPV), negative predictive value (NPV) and AUC (area under the receiver operating characteristic curve, ROC curve) was obtained.

Results: All assays had a high sensitivity for the detection of CIN2+. p16/Ki67 dual staining had similar sensitivity with hr-HPV test for CIN2+ detection ( $89.9 \%$ vs $93.9 \%, P=0.781)$, and had similar sensitivity with LBC test $(89.9 \%$ vs $82.7 \%, P=0.588)$. However, $\mathrm{p} 16 / \mathrm{Ki} 67$ dual staining had higher specificity than that of both hr-HPV test $(70.1 \%$ vs $25.5 \%, P<0.001)$ and LBC test $(70.1 \%$ vs $38.9 \%, P=0.002)$ for CIN2+ detection. p16/Ki67 dual staining had bigger AUC (0.80) than that of hr-HPV test (0.60) and LBC test (0.61), the $P$ value was $0.002,0.003$, respectively. The specificity of dual staining for CIN2+ detection in hr-HPV positive women was $70.1 \%$, which was higher than that of LBC test $(41.9 \%, P=0.020)$. Colposcopy referral rate of $\mathrm{p} 16 / \mathrm{Ki} 67$ was lower than that of both $\mathrm{hr}-\mathrm{HPV}$ and LBC $(\mathrm{P}=0.002,<0.001$, respectively). HPV infection was significantly associated with p16/Ki67 expression in all patients. p16/Ki67 expression in HPV16/18 and other 15 types of hr-HPV infection was significantly higher than negative hr-HPV infection, odds ratio (OR) was $12.16(95 \%$ confidence interval, $95 \% \mathrm{Cl}=5.82-25.41)$ and $2.65(95 \% \mathrm{Cl}=$ 1.27-5.51), respectively.

Conclusions: p16/Ki67 dual staining has a good specificity of high-grade cervical lesions detection and is a promising tool in triage of CIN2+ and hr-HPV positive population and avoid over diagnosis and treatment.

\section{Background}

Cervical cancer was the most common gynecological malignancies in women in developing countries and ranked third in cancer incidence throughout the world [1-2]. Recent global data estimate 527,624 new cases of cervical cancer annually and $84.2 \%$ of which occurred in developing countries [3]. Although the incidence of cervical cancer is high, the development of cancer is a slow process, usually develops from precancerous lesion, which called low-grade or high-grade squamous intraepithelial lesions (LSIL or HSIL) [4]. Early and efficiency detecting of LSIL or HSIL during the process of precancerous lesion can interrupt 
the invasive cancer sequelae and decrease the incidence of cervical cancer, which makes the screening of cervical cancer particularly important.

Multiple studies have documented that increasing incidence of human papillomavirus (HPV) infection leads to high incidence of cervical cancer among younger women because of the sexual behavior at an early age [5]. Studies have shown that the most common screening method was high-risk HPV (hr-HPV) test. The superior sensitivity of clinically validated hr-HPV test and high repeatability with acceptable negative predictive value makes this method widely accepted as the most important strategy for the cervical cancer screening [6-7]. However, because of the unsatisfactory performance of hr-HPV test, the Preventive Services Task Force screening guidelines of the United States prefer primary hrHPV test every 5 years for screening [8].

Another important screening tool is cytology based on the Papanicolaou (Pap) smear, which was in largescale use and reduced the cervical cancer incidence and mortality in many regions [7]. However, because of its low sensitivity for detecting cervical intraepithelial neoplasia grade 2 (CIN2) or higher lesion [4], clinicians prone to find other effective tools to screen the cervical cancer, one of whic was $\mathrm{p} 16 / \mathrm{Ki}-67$ dual staining.

The protein p16INK4a $(\mathrm{p} 16)$ is a cell-cycle regulatory protein which plays an important role in inducing cell-cycle arrest [9]. The over-expression of p16 histology specimens is considered as a good biomarker for detecting precancer lesions because it can deregulated HPV DNA E7 oncogenes expression and hence for transforming HPV infections [4]. Ki-67 is a cell proliferation marker which is widely used in evaluation of cervical epithelial lesions. The simultaneous detection of p16 and Ki67 can improve the sensitivity and specificity of lesions on cervix uteri, especially identification for cervical high-grade lesions [10]. However, the sensitivity and specificity of biomarkers which detected cervical lesions among younger women were not well documented. In this study, we evaluated the diagnostic accuracy of hr-HPV test, cytology and p16/Ki-67 dual staining in detection of HSIL in women aged $\leq 30$ years and explored the importance of p16/Ki-67 in triaging the cervical lesions.

\section{Methods}

\subsection{Study population}

This prospective study included all women attended at the gynecology department of Shunde Hospital of Guangzhou University of Chinese Medicine for first treating cervical lesions between 2018 January and 2019 December. The inclusion criteria considered: (1) aged $\leq 30$ years; (2) had sexual behavior; (3) nonpregnancy; (4) had no history of cervical surgery or chemo and/or radiotherapy for cervical malignant disease. The exclusion criteria was as follows: (1) had no hr-HPV test; (2) had no cytology test; (3) had no p16/Ki-67 dual staining test; (4) had no cervical histopathological diagnosis. A total of 336 women were enrolled in this study and cervical exfoliated cell specimens were collected and tested for hr-HPV, cytology and p16/Ki-67 immunocytochemistry. The Ethics Committee of Shunde Hospital of Guangzhou University of Chinese Medicine reviewed and approved of this study. 


\subsection{High-risk HPV detection}

High-risk HPV detection was performed using HPV DNA Array genotyping assay (Luminex Diagnostic Products). HPV DNA Array genotyping assay technique used fully automated sample preparation combined with multiplex real-time polymerase chain reaction (PCR) and associated techniques. It can detect genotyping 17 high risk $(16,18,31,33,35,39,45,51,52,56,58,59,66,68,82,53,26)$ and 10 low risk HPV types.

\subsection{Liquid-based cytology}

LBC technology was used to perform cytological detection. We used Thinprep method (Cytyc Corporation, Taikang, China) to make slides and stain by Papanicolaou method. The 2001 Bethesda Reporting System Criteria was used to report the diagnosis results [11].

\section{4 p16/ki-67 dual staining}

A second cytology slide was stained for p16 and Ki67 using CINtec PLUS cytology kit (Roche Diagnostic Products) according to the manufacturer's instruction. Positive of p16/ki-67 dual staining was defined as simultaneously brownish cytoplasmic immunostaining (p16) and red cytoplasmatic staining (Ki-67) in the same cell. Specimens without any double-stained cell were defined as negative of p16/ki-67 dual staining. Two researchers were interpreted the cytology results and if there was inconsistent result, the slide was reviewed by third researcher.

\subsection{Histopathology}

Colposcopy and cervical biopsy were performed by gynecologists. Cervical histopathological diagnosis was made by pathologists. Diagnosis results included inflammation, CIN1, CIN2, CIN3 and cervical cancer. CIN2, CIN3 and cervical cancer were considered as HSIL and higher lesions (CIN2+).

\subsection{Statistical analyses}

IBM SPSS Statistical Software (version 23.0) was used to calculate data. The sensitivity, specificity, positive predictive value (PPV), negative predictive value (NPV) and $95 \%$ confidence interval $(\mathrm{Cl})$ were calculated for pathology of of p16/Ki67 dual staining, hr-HPV test and cytology. Pearson $\chi 2$ and McNemar $\chi 2$ test were performed to compared different diagnosis methods. The area under the receiver operating characteristic curve (ROC) curve (AUC) were calculated and compared among three methods. A two-sided $\mathrm{P}<0.05$ indicated statistical significance.

\subsection{Ethics statement}

The study was approved by the Ethics Committee of Liuzhou Maternity and Child Healthcare Hospital, and informed consent was signed by guardian before the enrollment was performed. 


\section{Results}

\subsection{Study population}

A total of 21 patients were excluded in this study because of the following reasons: invaild hr-HPV test (12); invalid cytology test and results (5), lack of clear diagnosis (4). Among the final 336 women, 59 were diagnosed CIN1, 99 were diagnosed CIN2, 67 were diagnosed CIN3 and 13 were diagnosed cervical cancer according to histopathology results. There were 208 cases $(61.9 \%)$ showing positive of p16/Ki67 dual staining, 285 cases (84.8\%) showing positive of hr-HPV and 244 cases (72.6\%) showing positive of cytology (Table 1$)$.

Table $1 \mathrm{HPV}$, TCT and p16/Ki-67 dual staining in cervical lesions of women aged $\leq 35$ years. opathology Number (\%) HrHPV + HPV16/18 ASC-US + p16/Ki-67 + LBC +

\begin{tabular}{lllllll}
\hline ign lesion & $98(29.2)$ & 70 & 28 & 2 & 28 & 57 \\
1 & $59(17.6)$ & 47 & 27 & 15 & 19 & 39 \\
2 & $99(29.5)$ & 94 & 60 & 31 & 89 & 77 \\
3 & $67(19.9)$ & 64 & 49 & 18 & 59 & 61 \\
& $13(3.9)$ & 10 & 10 & 5 & 13 & 10 \\
l number & $336(100.0)$ & 285 & 174 & 71 & 208 & 244 \\
\hline
\end{tabular}

hr-HPV, high-risk human papillomavirus; HPV, human papillomavirus; ASC-US, atypical squamous cells of undetermined significance; LBC, liquid-based cytology; CIN, cervical intraepithelial neoplasia; CC, cervical cancer

\section{2 p16/Ki67 dual staining had higher specificity and bigger AUC to detect CIN2 or higher lesions}

The sensitivity, specificity, PPV, NPV and AUC of p16/Ki67 dual staining, hr-HPV test and cytology for HSIL and higher cervical lesions (CIN2+) were reported in Table 2. The sensitivity of p16/Ki67 dual staining, hr-HPV test and cytology for pathological diagnosis of CIN2+ was $89.9 \%, 93.9 \%$ and $82.7 \%$, respectively. The specificity of p16/Ki67 dual staining was $70.1 \%$, which was higher than that of hr-HPV test $(25.5 \%, P<0.001)$ and cytology test $(38.9 \%, P=0.002)$. The PPV of $\mathrm{p} 16 / \mathrm{Ki} 67$ dual staining was $77.4 \%$, which was higher than that of hr-HPV test $(58.9 \%, P<0.001)$ and cytology test $(60.7 \%, P<0.001)$. The AUC of $\mathrm{p} 16 / \mathrm{Ki} 67$ dual staining was 0.80 , which was bigger than that of hr-HPV test $(0.60, P=0.002)$ and cytology test $(0.61, P=0.003)$. Colposcopy referral rate of $\mathrm{p} 16 / \mathrm{Ki} 67$ dual staining for $\mathrm{CIN} 1+$ was lower than that of hr-HPV test $(49.7 \%$ vs $64.0 \%, P<0.001)$ and cytology test $(49.7 \%$ vs $61.6 \%, P<0.001)$.

Table 2 Detection of CIN2 and higher cervical lesions by three methods 


\begin{tabular}{llllll}
\hline & p16/Ki-67 & Hr-HPV & LBC & P1 & P2 \\
\hline tivity \% (95\% CI) & $89.9(85.5-$ & $93.9(90.3-$ & $82.7(77.1-$ & 0.781 & 0.588 \\
& $94.4)$ & $97.4)$ & $88.3)$ & & \\
ficity \% (95\% CI) & $70.1(62.8-$ & $25.5(18.6-$ & $38.9(31.1-$ & $<0.001$ & 0.002 \\
& $77.3)$ & $32.4)$ & $46.6)$ & & \\
\% (95\% CI) & $77.4(71.7-$ & $58.9(53.2-$ & $60.7(54.5-$ & $<0.001$ & $<0.001$ \\
& $83.1)$ & $64.7)$ & $66.8)$ & & \\
\% (95\% CI) & $85.9(79.8-$ & $78.4(66.7-$ & $66.3(56.5-$ & 0.712 & 0.217 \\
& $92.0)$ & $90.1)$ & $76.1)$ & & \\
(95\% CI) & $0.80(0.75-$ & $0.60(0.54-$ & $0.61(0.55-$ & 0.002 & 0.003 \\
& $0.85)$ & $0.66)$ & $0.67)$ & & \\
srral Rate \% (95\% & $49.7(44.3-$ & $64.0(58.8-$ & $61.6(56.4-$ & $<0.001$ & $<0.001$ \\
& $55.1)$ & $69.1)$ & $66.8)$ & & \\
\hline
\end{tabular}

P1, indicate the comparison between p16/Ki-67 and high-risk HPV test; P2, indicate the comparison between p16/Ki67 and LBC; hr-HPV, high-risk human papillomavirus; LBC, liquidbased cytology; CI, confidence interval; PPV, positive predictive value; NPV, negative predictive value; AUC, area under ROC curve; † colposcopy referral rate of CIN1 and higher cervical lesions

\section{3 p16/Ki67 dual staining had high specificity and bigger AUC to detect CIN2 or higher lesions in women with positive hr-HPV test}

The sensitivity, specificity, PPV, NPV and AUC of p16/Ki67 dual staining, HPV16/18 test and cytology for CIN2+ and colposcopy referral rate were demonstrated in Table 3. The sensitivity of p16/Ki67 dual staining, HPV16/18 test and cytology for pathological diagnosis of CIN2+ was $89.9 \%, 70.8 \%$ and $81.0 \%$, respectively. The specificity of p16/Ki67 dual staining was $70.1 \%$, which was higher than that of hr-HPV test $(53.0 \%, P=0.007)$ and cytology test $(41.9 \%, P=0.002)$. The AUC of $\mathrm{p} 16 / \mathrm{Ki} 67$ dual staining was 0.80 , which was bigger than that of HPV $16 / 18$ test $(0.62, P=0.005)$ and cytology test $(0.61, P=0.003)$. All cases with positive hr-HPV need colposcopy referral, but in patients with positive hr-HPV test, the colposcopy referral rate of specimens with p16/Ki67 dual staining (78.6\%) was similar to that by cytology $(74.7 \%)$, but higher than that with HPV $16 / 18$ positive test $(60.1 \%, P=0.010)$.

Table 3 Detection of CIN2 and higher cervical lesions in HR-HPV positive patients 


\begin{tabular}{llllll}
\hline & $\mathrm{p} 16 / \mathrm{Ki}-67$ & $\mathrm{LBC}$ & HPV16/18 & P1 & P2 \\
\hline sitivity \% (95\% CI) & $89.9(85.3-$ & $81.0(75.0-$ & $70.8(63.9-$ & 0.515 & 0.146 \\
& $94.5)$ & $87.0)$ & $77.8)$ & & \\
cificity \% (95\% CI) & $70.1(61.7-$ & $41.9(32.8-$ & $53.0(43.8-$ & $<0.001$ & 0.007 \\
& $78.5)$ & $51.0)$ & $62.2)$ & & \\
\% (95\% CI) & $81.2(75.5-$ & $66.7(60.1-$ & $68.4(61.4-$ & 0.206 & 0.289 \\
& $86.9)$ & $73.2)$ & $75.4)$ & & \\
I\% (95\% CI) & $82.8(75.3-$ & $60.5(49.6-$ & $55.9(46.5-$ & $<0.001$ & $<0.001$ \\
& $90.4)$ & $71.4)$ & $65.2)$ & & \\
ว (95\% CI) & $0.80(0.74-$ & $0.61(0.55-$ & $0.62(0.55-$ & 0.003 & 0.005 \\
& $0.86)$ & $0.68)$ & $0.69)$ & & \\
ərral Rate \% (95\% & $78.6(72.3-$ & $74.7(67.9-$ & $60.1(52.6-$ & 0.868 & 0.010 \\
& $84.8)$ & $81.5)$ & $67.6)$ & & \\
\hline
\end{tabular}

$\mathrm{P} 1$, indicate the comparison between p16/Ki-67 and LBC; P2, indicate the comparison between p16/Ki-67 and HPV16/18; LBC, liquid-based cytology; HPV, human papillomavirus; CI, confidence interval; PPV, positive predictive value; NPV, negative predictive value; AUC, area under ROC curve

\subsection{HPV infection was significantly associated with $\mathrm{p} 16 / \mathrm{Ki} 67$ expression in all patients}

To explore the association between p16/Ki67 dual staining and hr-HPV infection, we divided the patients into three groups according to HPV infection status. HPV infection was significantly associated with p16/Ki67 expression in all patients (Table 4). p16/Ki67 expression in HPV16/18 and other 15 types of hrHPV infection was significantly higher than negative hr-HPV infection, odds ratio (OR) was 12.16 (95\% confidence interval, $95 \% \mathrm{Cl}=5.82-25.41)$ and $2.65(95 \% \mathrm{Cl}=1.27-5.51)$, respectively. In patients with CIN2+, p16/Ki67 expression in HPV16/18 was associated with HPV 16/18 infection, with OR was 6.56 $(95 \% \mathrm{Cl}=2.47-17.40)$. Other 15 types of hr-HPV infection was also associated with higher risk of p16/Ki67 expression than hr-HPV negative patients in CIN2+ cervical lesions, with OR was $7.67(95 \% \mathrm{Cl}=$ 1.51-38.98).

Table 4 Association between p16/Ki67 and hr-HPV test in different histology categories 


\begin{tabular}{|c|c|c|c|c|}
\hline hr-HPV categories & $\begin{array}{l}\mathrm{P} 16 / \mathrm{Ki67}+ \\
(\mathrm{n}, \%)\end{array}$ & $\begin{array}{l}\mathrm{P} 16 / \mathrm{Ki} 67 \\
(\mathrm{n}, \%)\end{array}$ & OR(95\%CI) & $P$ \\
\hline Hr-HPV negative $(n=50)$ & $13(26.0)$ & $37(74.0)$ & Ref & \\
\hline $\begin{array}{l}\text { Other } 15 \text { types of } \mathrm{hr} \text {-HPV } \\
\text { positive }(\mathrm{n}=112)\end{array}$ & $54(48.2)$ & $58(51.5)$ & $\begin{array}{l}2.65(1.27- \\
5.51)\end{array}$ & $<0.001$ \\
\hline $\begin{array}{l}\text { HPV } 16 / 18 \quad \text { positive } \\
(\mathrm{n}=174)\end{array}$ & $141(81.0)$ & $33(19.0)$ & $\begin{array}{l}12.16(5.82- \\
25.41)\end{array}$ & $<0.001$ \\
\hline Hr-HPV negative $(n=40)$ & $7(17.5)$ & $33(82.5)$ & Ref & \\
\hline $\begin{array}{l}\text { Other } 15 \text { types of } \mathrm{hr} \text {-HPV } \\
\text { positive }(\mathrm{n}=62)\end{array}$ & $8(12.9)$ & $54(87.1)$ & $\begin{array}{l}0.69(0.23- \\
2.10)\end{array}$ & 0.524 \\
\hline HPV $16 / 18$ positive $(n=55)$ & $32(58.2)$ & $23(41.8)$ & $\begin{array}{l}6.56(2.47- \\
17.40)\end{array}$ & $<0.001$ \\
\hline Hr-HPV negative $(n=10)$ & $6(60.0)$ & $4(40.0)$ & Ref & \\
\hline $\begin{array}{l}\text { Other } 15 \text { types of hr-HPV } \\
\text { positive }(n=50)\end{array}$ & $46(92.0)$ & $4(8.0)$ & $\begin{array}{l}7.67(1.51- \\
38.98)\end{array}$ & 0.014 \\
\hline $\begin{array}{l}\mathrm{HPV} \\
(\mathrm{n}=119)\end{array}$ & $109(91.6)$ & $10(8.4)$ & $\begin{array}{l}7.27(1.75- \\
30.10)\end{array}$ & 0.006 \\
\hline
\end{tabular}

Abbreviations: OR, Odds ratio; CI, confidence interval; CIN2+, cervical intraepithelial neoplasia grade 2 or higher lesions. Other $15 \mathrm{hr}$-HPV positive: positive for any of the $15 \mathrm{HPV}$ types (31, $33,35,39,45,51,52,56,58,59,66,68,82,53,26)$, and negative for HPV16/18.

\section{Discussion}

Our study demonstrated that p16/Ki67 dual staining, hr-HPV and cytology had high sensitivity to detect high grade cervical lesions (CIN2+), which was in keeping with other reported studies $[9,12]$. Although some of the studies reported that the specificity of p16/Ki67 dual staining to detect CIN2+ was high, but there was no difference between p16/Ki67 dual staining and hr-HPV test [13]. Our study reported that the specificity of p16/Ki67 dual staining was significantly higher than that of hr- HPV and cytology test. At the same time, when we considered the histopathology results as the gold standard to detect the cervical lesions, p16/Ki67 dual staining had bigger AUC than hr-HPV and cytology test, which indicated that p16/Ki67 dual staining had better distinction without reducing the sensitivity for detecting high grade cervical lesions. 
In our study, all patients with a positive result in the hr-HPV test or with CIN1 or higher lesion by cytology would refer to colposcopy and biopsy, allowing the performance of p16/Ki67 to triage women with CIN1 or higher lesion. Because less than $5 \%$ of CIN1 would progress and more than $30 \%$ of CIN1 regress within 1 year [12], so high sensitivity and specificity of detection method would lead to lower probability to refer to colposcopy especially in the women who have low risk of underlying high grade lesions. Our study reported that p16/Ki67 dual staining had lower referral rate to colposcopy than hr- HPV and cytology test to detect CIN1 and higher cervical lesions, which indicated that p16/Ki67 was an important triage tool for detecting cervical lesions to reduce the number of unnecessary colposcopy referrals and avoid excessive diagnosis and treatment.

Our study reported that p16/Ki67 dual staining, HPV 16/18 and cytology had similar sensitivity to detect CIN2+ cervical lesions in women with hr-HPV positive, which was in consistent with other studies [14]. Although there was a high sensitivity of hr-HPV test for detecting abnormal cervical lesions, but this biomarker could not differentiate between transient infection and persistent infection [15]. In most cases, patients had hr-HPV test positive results referral to colposcopy which may increase the referral rate and waste of medical resources because of the low specificity of hr-HPV test to detect CIN2+ [16]. Some studies reported that in order to reduce excessive diagnosis when patient had hr-HPV test result, a useful triage tool must be lunched [12]. Our study reported that p16/Ki67 dual staining had high sensitivity and the specificity of which was higher than that of HPV16/18 or cytology in patients with HPV positive patients to detect CIN2 and higher cervical lesions, which was in keeping with other literature [12]. The above studies indicated that when triage of patients with hr-HPV positive test, p16/Ki67 dual staining can improve the specificity of CIN2+ detection. which thereby can reduce referral rate to colposcopy and avoid over-waste of medical resource.

With the grade of cervical lesions increased gradually, the detection rate of p16/Ki-67 increased accordingly, which was in keeping with previous reports [12,17]. When cervical cells are transformed through hr-HPV infections, the inactivation of the retinoblastoma protein (pRb) by the HPV E7 oncogenic protein E7 would lead to over-expression of p16 in cervical dysplasia [18], which can serve as an useful biomarker of precancer and cancer in cervical lesions. Ki-67 protein plays an important role in regulation of cell cycle and can serve as a biomarker for cell proliferation in both normal and abnormal cells [19]. In this study, a significant association between p16/Ki67 dual staining positive and the hr-HPV test positive was reported, and 11.16-fold higher in the HPV 16 / 18 infection than that in the group of hr-HPV negative was observed in all of the abnormal lesions. When it comes to the higher lesions (CIN2+), the risk of overexpression of $\mathrm{p} 16 / \mathrm{Ki} 67$ was $7.27(95 \% \mathrm{Cl}=1.75-30.10, P=0.006)$ in the HPV $16 / 18$ positive group compared to hr-HPV negative group, which indicated that p16/Ki67 over-expression within the same cell could be a good marker of deregulation of the cell cycle and a transforming HPV 16/18 infection which may progress to abnormal cervical lesions.

\section{Conclusions}


In conclusion, our study found that $\mathrm{p} 16 / \mathrm{Ki} 67$ dual staining could be use as a promising tool to identify cervical precancer and cancer based on its high specificity and big AUC. It could be used for triaging women with hr-HPV positive whom were further referred to colposcopies. p16/Ki67 dual staining could improve the sensitivity and specificity, reduce patient referral, and avoid unnecessary colposcopy compared with the hr-HPV and cytological triaging in women aged $\leq 30$ years.

\section{Declarations}

\section{Ethics approval and consent to participate}

This study was approved by the Institutional Review Board of Liuzhou Maternity and Child Healthcare Hospital.

\section{Consent to publish}

Not applicable.

\section{Availability of data and materials}

The data and materials is list as appendix.

\section{Competing interests}

All the authors including Eric McGrath declare that they have no competing interests.

\section{Funding}

This manuscript was funded by The Key Project of Science and Technology in Guangdong Provincial Hospital of Traditional Chinese Medicine (YN2016ZD03), Sino-National Natural Science Foundation (No.81760355) and Liuzhou Science and Technology Bureau Project (2014J030418, 2017BH20310). The funders had no role in study design, data collection and analysis, decision to publish, or preparation of the manuscript.

\section{Authors' contributions}

Conceived and designed the experiments: Xingmin Wang, Xindan Wang and Jianhua Xu. Performed the experiments: Jianhua Xu. Analyzed the data: Ping Wu, Jinjian Fu and Guixian Yi. Contributed reagents/materials/analysis tools: Ping Wu, Jinjian Fu and Guixian Yi. Wrote and revise the manuscript: Xingmin Wang, Xindan Wang, Guixian Yi and Jianhua Xu.

\section{Acknowledge}

Not applicable.

\section{Author's information}


${ }^{1}$ Department of Pathology, Liuzhou Maternity and Child Health Care Hospital, Liuzhou, Guangxi, China (8wangxingmin@163.com);

${ }^{2}$ Department of Gynecologic Oncology, Liuzhou Maternity and Child Health Care Hospital, Liuzhou, Guangxi, China (wangxindan771124@163.com);

${ }^{3}$ Department of Pharmacy, Chengdu First People's Hospital/ Chengdu Integrated TCM \& Western Medicine Hospital, Chengdu, Sichuan, China (1601820859@qq.com);

${ }^{4}$ Department of Clinical Laboratory, Liuzhou Maternity and Child Health Care Hospital, Liuzhou, Guangxi, China(fujinjianaa@126.com);

${ }^{5}$ Department of Gynecologic Oncology, Dongguan Humen Hospital of Chinese Medicine, Dongguan, Guangdong, China(269440812@qq.com)

${ }^{6}$ Department of Oncology and Molecular Biology Laboratory, Shunde Hospital of Guangzhou University of Chinese Medicine, Foshan ,Guangdong, China (jhxu1976@126.com)

\section{References}

1. Moshkovich O, Lebrun-Harris L, Makaroff L, et al. Challenges and Opportunities to Improve Cervical Cancer Screening Rates in US Health Centers through Patient-Centered Medical Home Transformation. Adv Prev Med. 2015;2015:182073.

2. Senkomago V, Henley SJ, Thomas CC, Mix JM, Markowitz LE, Saraiya M. Human PapillomavirusAttributable Cancers - United States, 2012-2016. MMWR Morb Mortal Wkly Rep. 2019;68(33):724728.

3. Torre LA, Bray F, Siegel RL, Ferlay J, Lortet-Tieulent J, Jemal A. Global cancer statistics, 2012. CA Cancer J Clin. 2015;65(2):87-108.

4. Zhu Y, Ren C, Yang L, Zhang X, Liu L, Wang Z. Performance of p16/Ki67 immunostaining, HPV E6/E7 mRNA testing, and HPV DNA assay to detect high-grade cervical dysplasia in women with ASCUS. BMC Cancer. 2019;19(1):271.

5. Shrestha AD, Neupane D, Vedsted P, Kallestrup P. Cervical Cancer Prevalence, Incidence and Mortality in Low and Middle Income Countries: A Systematic Review. Asian Pac J Cancer Prev. 2018;19(2):319324.

6. Saslow D, Solomon D, Lawson HW, et al. American Cancer Society, American Society for Colposcopy and Cervical Pathology, and American Society for Clinical Pathology screening guidelines for the prevention and early detection of cervical cancer. Am J Clin Pathol. 2012;137(4):516-542.

7. Stoler MH, Baker E, Boyle S, et al. Approaches to triage optimization in HPV primary screening: Extended genotyping and p16/Ki-67 dual-stained cytology -Retrospective insights from ATHENA. Int J Cancer. 2020;146(9):2599-2607. 
8. US Preventive Services Task Force, Curry SJ, Krist AH, et al. Screening for Cervical Cancer: US Preventive Services Task Force Recommendation Statement. JAMA. 2018;320(7):674-686.

9. Qian QP, Zhang X, Ding B, et al. Performance of P16/Ki67 dual staining in triaging hr-HPV-positive population during cervical Cancer screening in the younger women. Clin Chim Acta. 2018;483:281285.

10. Areán-Cuns C, Mercado-Gutiérrez M, Paniello-Alastruey I, et al. Dual staining for $p 16 / \mathrm{Ki} 67$ is a more specific test than cytology for triage of HPV-positive women. Virchows Arch. 2018;473(5):599-606.

11. Solomon D, Davey D, Kurman R, et al. The 2001 Bethesda System: terminology for reporting results of cervical cytology. JAMA. 2002;287(16):2114-2119.

12. Zhang R, Ge X, You K, Guo Y, Guo H, Wang Y, Geng L. p16/Ki67 dual staining improves the detection specificity of high-grade cervical lesions. J Obstet Gynaecol Res. 2018 Nov;44(11):2077-2084.

13. Kisser A, Zechmeister-Koss I. A systematic review of p16/Ki-67 immuno-testing for triage of low grade cervical cytology. BJOG. 2015 Jan;122(1):64-70.

14. Jiang MY, Wu Z, Li T, et al. Performance of HPV Genotyping Combined with p16/Ki-67 in Detection of Cervical Precancer and Cancer Among HPV-Positive Chinese Women. Cancer Prev Res (Phila). 2020 Feb;13(2):163-172.

15. Nobbenhuis MA, Helmerhorst TJ, van den Brule AJ, et al. Cytological regression and clearance of high-risk human papillomavirus in women with an abnormal cervical smear. Lancet. 2001 Nov 24;358(9295):1782-3.

16. Stoler MH, Wright TC Jr, Sharma A, Apple R, Gutekunst K, Wright TL; ATHENA (Addressing THE Need for Advanced HPV Diagnostics) HPV Study Group. High-risk human papillomavirus testing in women with ASC-US cytology: results from the ATHENA HPV study. Am J Clin Pathol. 2011 Mar;135(3):46875 .

17. Yu LL, Chen W, Lei XQ, et al. Evaluation of p16/Ki-67 dual staining in detection of cervical precancer and cancers: a multicenter study in China. Oncotarget. 2016 Apr 19;7(16):21181-9.

18. von Knebel Doeberitz M. New markers for cervical dysplasia to visualise the genomic chaos created by aberrant oncogenic papillomavirus infections. Eur J Cancer. 2002 Nov;38(17):2229-42.

19. Scholzen T, Gerdes J. The Ki-67 protein: from the known and the unknown. J Cell Physiol. 2000 Mar;182(3):311-22. 